Hydrol. Earth Syst. Sci., 17, 4701-4712, 2013

www.hydrol-earth-syst-sci.net/17/4701/2013/

doi:10.5194/hess-17-4701-2013

(c) Author(s) 2013. CC Attribution 3.0 License.

\title{
Rainfall estimation using moving cars as rain gauges - laboratory experiments
}

\author{
E. Rabiei ${ }^{1}$, U. Haberlandt ${ }^{1}$, M. Sester ${ }^{2}$, and D. Fitzner ${ }^{2}$ \\ ${ }^{1}$ Institute of Water Resources Management, Hydrology and Agricultural Hydraulic Engineering, Leibniz Universität \\ Hannover, Hanover, Germany \\ ${ }^{2}$ Institute of Cartography and Geoinformatics, Leibniz Universität Hannover, Hanover, Germany \\ Correspondence to: E. Rabiei (rabiei@iww.uni-hannover.de)
}

Received: 18 February 2013 - Published in Hydrol. Earth Syst. Sci. Discuss.: 4 April 2013

Revised: 4 September 2013 - Accepted: 25 October 2013 - Published: 28 November 2013

\begin{abstract}
The spatial assessment of short time-step precipitation is a challenging task. Low density of observation networks, as well as the bias in radar rainfall estimation motivated the new idea of exploiting cars as moving rain gauges with windshield wipers or optical sensors as measurement devices. In a preliminary study, this idea has been tested with computer experiments (Haberlandt and Sester, 2010). The results have shown that a high number of possibly inaccurate measurement devices (moving cars) provide more reliable areal rainfall estimations than a lower number of precise measurement devices (stationary gauges). Instead of assuming a relationship between wiper frequency $(W)$ and rainfall intensity $(R)$ with an arbitrary error, the main objective of this study is to derive valid $W-R$ relationships between sensor readings and rainfall intensity by laboratory experiments. Sensor readings involve the wiper speed, as well as optical sensors which can be placed on cars and are usually made for automating wiper activities. A rain simulator with the capability of producing a wide range of rainfall intensities is designed and constructed. The wiper speed and two optical sensors are used in the laboratory to measure rainfall intensities, and compare it with tipping bucket readings as reference. Furthermore, the effect of the car speed on the estimation of rainfall using a car speed simulator device is investigated. The results show that the sensor readings, which are observed from manual wiper speed adjustment according to the front visibility, can be considered as a strong indicator for rainfall intensity, while the automatic wiper adjustment show weaker performance. Also the sensor readings from optical sensors showed promising results toward measuring rainfall
\end{abstract}

rate. It is observed that the car speed has a significant effect on the rainfall measurement. This effect is highly dependent on the rain type as well as the windshield angle.

\section{Introduction}

Accurate spatial precipitation assessment for short time steps has been of research interest for some time. However, due to its high variability in time and space, rainfall observation is still a challenging task. For instance, Schilling (1991) has discussed the need for higher resolution data with 1 min time resolution and $1 \mathrm{~km}^{2}$ spatial resolution in urban hydrology. Recent developments regarding modern instrumentation of recording gauges as well as the implementation of weather radar revealed a bright prospect for estimation of areal precipitation in short time steps. Recording gauges provide valuable point rainfall depths, but these are still often poor in density. Weather radar has become an essential source for rainfall estimation. For example, Chandrasekar et al. (2012) showed the importance of high-resolution rainfall data using a X-band radar network for urban flash flood application. Despite its high spatial resolution, radar data has often a large space-time variable bias in rainfall estimation (Javier et al., 2007). There are several innovative methods which discuss new measurement techniques for rainfall intensity such as satellites (Diop and Grimes, 2003), microwave links (Upton et al., 2005), and acoustic rain gauges (de Jong, 2010). Leijnse et al. (2007) and Messer et al. (2006) are the pioneers in using radio links from cellular communication 
networks for rainfall measurement purposes. This is quite a new way of measuring rainfall and has been under study by several researchers recently (Overeem et al., 2013; Zinevich et al., 2009). Most of the mentioned methods seek to use alternative devices which were intended originally for other purposes. De Jong (2010) has also developed a low cost disdrometer to make measuring rainfall affordable with a very high spatial and temporal resolution.

The combination of data from different sources can improve the estimation of areal rainfall. For instance Haberlandt (2007) and Verworn and Haberlandt (2010) implemented kriging with external drift in order to combine radar data with rain gauge network data. Ehret (2002) applied another method for merging radar data with rain gauge data, called conditional merging. Other approaches for merging radar data with rain gauge data were suggested by Erdin et al. (2012) and Vogl et al. (2012).

The idea of using moving cars as rainfall measurement devices was proposed for the first time by Haberlandt and Sester (2010). They use wiper speed $(W)$ as an indication of rainfall rate $(R)$ by applying a hypothetical $W-R$ relationship with an assumption about the rainfall rate estimation error. A traffic model has been applied to generate car trajectories on roads in a river basin. Radar data has been used as reference rainfall to evaluate the work. The rain rate for rain gauges and moving cars has been extracted from the radar data. Afterwards, the results of assessing areal rainfall by implementing ordinary kriging for rain gauges and indicator kriging for moving cars have been compared. These results show that a large number of inaccurate measurement devices would improve the spatial precipitation assessment in comparison to a couple of accurate devices. Besides, this new rainfall information will provide a good possibility to use this data for merging with other sources of data like radar or station data.

The main objective of this study is to develop and analyze the relationships between sensor readings $(W)$ and rainfall intensity $(R)$ by laboratory experiments. Sensor readings in this paper involve wiper speed, which is controlled either manually by a driver or automatically by optical sensors, as well as signals from optical sensors which can be placed on cars and are designed to automate the wiper activity. Within an experimental setup the relevant sensor reading uncertainties are to be investigated. For that reason a rainfall simulator with the ability to produce a wide range of rain intensities is designed and constructed. Rain simulators are a subject of different studies, for example, erosion (Fiener et al., 2011), agriculture, horticulture, hydrology, etc. Soil erosion experiments mainly use rain simulators which aim to reproduce, as near as possible, the properties of natural rain (Salles and Poesen, 1999). The rain simulator used in this study should have the capability of producing different rain intensities with homogeneous distribution over the desired area as well as replicating the properties of natural rain. Analyses of rainfall measured by car sensors are accomplished considering a tipping bucket as reference device. There are many different

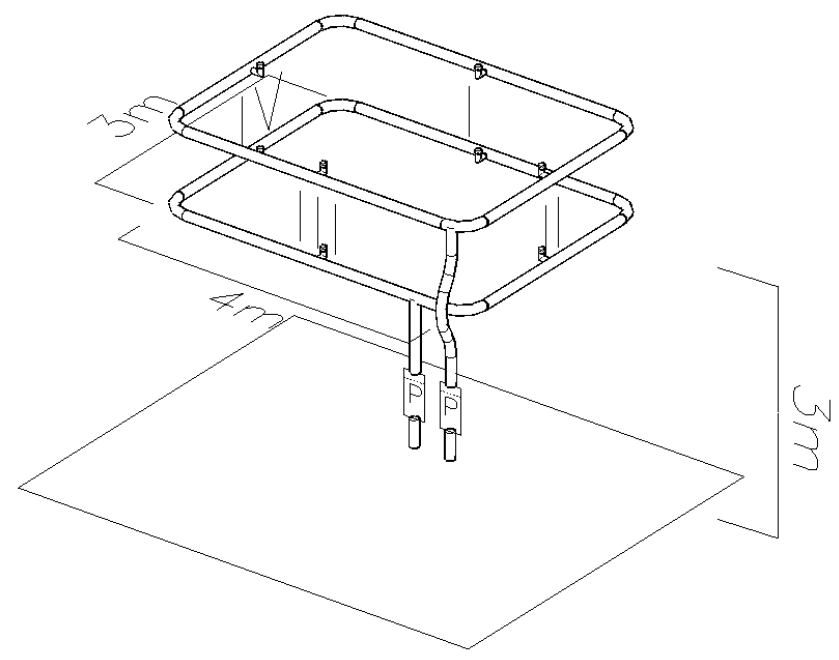

Fig. 1. Rain simulator, two layers with 8 nozzles.

environmental factors influencing the estimation of rainfall by cars in nature like car speed, wind speed, wind direction, windshield angle, etc. In this study, only the influence of the car speed on the estimation of rainfall is investigated with the help of a special car speed simulator.

The paper is organized as follows. Section two describes the rainfall simulator and the way it is designed. The description of the rainfall measurement devices and their functionality are provided in the third section. Section four discusses the results including the analyses of the produced rainfall and the derived $W-R$ relationships. The last section presents a summary and conclusion.

\section{Rainfall simulator - sprinkler irrigation system}

Considering the addressed purposes of the study, a wide range of rainfall intensities needed to be produced by a rainfall simulator. The points guiding the design of the system are (1) producing homogeneous rainfall in the laboratory, and (2) the ability of testing cars with measurement devices under different rain intensities. According to the design principles of the sprinkler irrigation system, given in FAO (Phocaides, 2000) or other handbooks, sprinkler spacing depends on the wetted diameter produced by each specific nozzle. Figure 1 shows the design of the rain simulator used for the laboratory experiments. It consists of two layers that have the capacity of positioning eight nozzles in total. All the measurement devices as well as the tested cars are placed under the rain simulator, which has a height of approximately $3 \mathrm{~m}$ from the ground. "P" in Fig. 1 shows the pressure controller which controls each layer's pressure. Considering the specifications for each nozzle provided by the manufacturer, the design of the rain simulator is based on a pressure of 2 bars and neglects head losses in pipes. To reproduce a larger range 
of rain intensities, pressures of 1 bar and 2.5 bars are also applied.

Producing different rain intensities is achieved by activating different sets of nozzles and applying different pressures on the nozzles. Table 1 presents detailed information about the three different nozzle models used in this study regarding their mean rainfall intensity and the maximum wetted radius under different pressures. It should be pointed out that in contrast to natural events, where the environmental factors influence the rain drops and fast rain rate variations can occur in a short period of time, the rainfall produced in the laboratory is constant over a certain time.

The numbers from I to IV in Fig. 1 shows the four spots available for placing the nozzles on each layer. Considering the water distribution pattern of the nozzles and the goal to produce homogeneous rainfall, the positions for the different nozzle types can be selected. Taking into account the mentioned factors and principles for sprinkler irrigation design, Table 2 shows different combinations of the nozzles used in this study. Altogether 8 classes of nozzle combinations are defined.

In Table 3 the Cartesian product of the set of pressures with the set of different nozzle combinations is given, excluding duplicates, which results in 39 pairs. Due to the capacity of the pump, only 32 pairs are applied. The stars in Table 3 show the sets where the demand is higher than the pump capacity. The rainfall intensities given in Table 3 for the 32 cases are measured with the tipping bucket reference device and cover a range between 9.2 and $98.1 \mathrm{~mm} \mathrm{~h}^{-1}$. For instance, the lowest produced rainfall intensity of $9.2 \mathrm{~mm} \mathrm{~h}^{-1}$ belongs to the nozzle combination class 1 applying a pressure of 1 bar using 2 nozzles of the type S-16A on positions I and III on the 1st layer. The highest rainfall intensity of $98.1 \mathrm{~mm} \mathrm{~h}^{-1}$ belongs to the nozzle combination class 5 with an application of 2.5 bar pressure using 8 nozzles of the type S-8A on positions I, II, III and IV for both layers.

Most of the rain simulators are not able to generate low rainfall intensities, for example, Sharpley and Kleinman (2003) were also able to produce rainfall intensities starting from $17.0 \mathrm{~mm} \mathrm{~h}^{-1}$. The generation of rainfall intensities lower than $9.2 \mathrm{~mm} \mathrm{~h}^{-1}$ in the laboratory is hardly possible because available nozzles providing uniform rainfall distribution usually cannot generate lower intensities. However, given that the application is intended primarily for flood producing situations, this lower rainfall intensity limit is considered sufficient for this initial study.

The following analyses are performed using a constant rainfall intensity produced by the sprinklers in a time period of $15 \mathrm{~min}$ for all the possible 32 cases.

\section{Rainfall measurement devices}

Two kinds of measurement instruments are used in the laboratory, a reference gauge and devices which are meant for
Table 1. Specification of the nozzles used in this study, given by the manufacturer.

\begin{tabular}{lccc}
\hline $\begin{array}{c}\text { Nozzle } \\
\text { model }\end{array}$ & $\begin{array}{c}\text { Pressure } \\
{[\text { bar }]}\end{array}$ & $\begin{array}{c}\text { Precipitation } \\
{\left[\mathrm{mm} \mathrm{h}^{-1}\right]}\end{array}$ & $\begin{array}{c}\text { Radius } \\
{[\mathrm{m}]}\end{array}$ \\
\hline \multirow{3}{*}{ S-8A } & 1.37 & 62.2 & 2.13 \\
& 2.06 & 50.8 & 2.43 \\
& 2.41 & 52.1 & 2.43 \\
\hline \multirow{3}{*}{ S-16A } & 1.37 & 15 & 4.57 \\
& 2.06 & 18.3 & 4.88 \\
& 2.41 & 17.8 & 5.18 \\
\hline \multirow{3}{*}{ 8A } & 1.37 & 45.2 & 2.13 \\
& 2.06 & 42.9 & 2.43 \\
& 2.41 & 36.8 & 2.74 \\
\hline
\end{tabular}

rainfall measurement with cars. The reference gauge provides the reference rainfall $(R)$ in relation to the car sensor readings $(W)$. The rainfall measurement devices are explained in the following.

\subsection{Reference gauge}

\section{Tipping bucket}

One of the most common devices of measuring rainfall depths is the tipping bucket rain gauge. Although the device is widely used for different purposes, it is subject to systematic and random instrumental errors (Ciach, 2003). However, wind as the most important factor influencing the measurement accuracy has no relevance in the laboratory. The tipping bucket used in this study has a minimum measurement resolution of $0.1 \mathrm{~mm}$ rainfall. The size of the bucket and rain intensity are the critical factors in estimating the rainfall for low intensities (Lanza et al., 2005). Since, in this study, only higher and constant rainfall intensities are applied in the laboratory, these uncertainties can be neglected. However, they are very critical for field measurements with finer temporal resolutions. So, it is suggested to implement, instead, more accurate rainfall measurement devices such as weighing rain gauges, as the reference for field experiments.

\subsection{Sensors considered for rainfall measurement by cars}

\subsubsection{Wiper frequency analysis}

The initial idea was to use the windshield wiper frequency as an indicator for rainfall intensity. The main goal here is to find a relationship between wiper speed $(W)$ and rainfall intensity $(R)$. This relationship is determined with the help of a stationary car placed under the rain simulator. Each car has a specific protocol for the wiper system, but the wiper systems are, in general, similar. The results of one car as a representative are presented in the following. 
Table 2. Different nozzle combinations implemented in this study.

\begin{tabular}{llcccccccc}
\hline & & \multicolumn{7}{c}{ Class } \\
\cline { 2 - 9 } & & 1 & 2 & 3 & 4 & 5 & 6 & 7 & 8 \\
\hline 1st & I & S-16A & S-16A & S-8A & S-8A & S-8A & $8 \mathrm{~A}$ & $8 \mathrm{~A}$ & $8 \mathrm{~A}$ \\
layer & II & - & - & S-8A & S-8A & S-8A & $8 \mathrm{~A}$ & $8 \mathrm{~A}$ & $8 \mathrm{~A}$ \\
& III & S-16A & S-16A & S-8A & S-8A & S-8A & $8 \mathrm{~A}$ & $8 \mathrm{~A}$ & $8 \mathrm{~A}$ \\
& IV & - & - & S-8A & S-8A & S-8A & $8 \mathrm{~A}$ & $8 \mathrm{~A}$ & $8 \mathrm{~A}$ \\
\hline \multirow{2}{*}{ 2nd } & I & - & S-16A & - & S-16A & S-8A & - & S-16A & $8 \mathrm{~A}$ \\
layer & II & - & - & - & - & S-8A & - & - & $8 \mathrm{~A}$ \\
& III & - & S-16A & - & S-16A & S-8A & - & S-16A & $8 \mathrm{~A}$ \\
& IV & - & - & - & - & S-8A & - & - & $8 \mathrm{~A}$ \\
\hline
\end{tabular}

Table 3. Applied pressures and corresponding produced rain intensities.

\begin{tabular}{|c|c|c|c|c|c|c|c|c|c|}
\hline & & \multicolumn{8}{|c|}{ Class } \\
\hline & & 1 & 2 & 3 & 4 & 5 & 6 & 7 & 8 \\
\hline & $\begin{array}{l}\mathrm{P} \\
{[\text { bar }]}\end{array}$ & $\begin{array}{c}\mathrm{PCP} \\
{\left[\mathrm{mm} \mathrm{h}^{-1}\right]}\end{array}$ & $\begin{array}{c}\mathrm{PCP} \\
{\left[\mathrm{mm} \mathrm{h}^{-1}\right]}\end{array}$ & $\begin{array}{c}\mathrm{PCP} \\
{\left[\mathrm{mm} \mathrm{h}^{-1}\right]}\end{array}$ & $\begin{array}{c}\mathrm{PCP} \\
{\left[\mathrm{mm} \mathrm{h}^{-1}\right]}\end{array}$ & $\begin{array}{c}\mathrm{PCP} \\
{\left[\mathrm{mm} \mathrm{h}^{-1}\right]}\end{array}$ & $\begin{array}{c}\mathrm{PCP} \\
{\left[\mathrm{mm} \mathrm{h}^{-1}\right]}\end{array}$ & $\begin{array}{c}\mathrm{PCP} \\
{\left[\mathrm{mm} \mathrm{h}^{-1}\right]}\end{array}$ & $\begin{array}{c}\mathrm{PCP} \\
{\left[\mathrm{mmh}^{-1}\right]}\end{array}$ \\
\hline $\begin{array}{l}1 \text { st } \\
\text { layer }\end{array}$ & 1 & 9.2 & - & 12.8 & - & - & 24.4 & - & - \\
\hline $\begin{array}{l}1 \text { st } \\
\text { layer }\end{array}$ & 2 & 16.8 & - & 37.7 & - & - & 34.4 & - & - \\
\hline $\begin{array}{l}\text { 1st } \\
\text { layer }\end{array}$ & 2.5 & 20.4 & - & 55.2 & - & - & 48.4 & - & - \\
\hline $\begin{array}{l}\text { 1st L. } \\
\text { 2nd L. }\end{array}$ & $1 \times 1$ & - & 14.4 & - & 11.6 & 40.7 & - & 33.4 & 60 \\
\hline $\begin{array}{l}\text { 1st L. } \\
\text { 2nd L. }\end{array}$ & $1 \times 2$ & - & 15.2 & - & 20.4 & 42 & - & 39.2 & $*$ \\
\hline $\begin{array}{l}\text { 1st L. } \\
\text { 2nd L. }\end{array}$ & $1 \times 2.5$ & - & 17.6 & - & 20.4 & 59.2 & - & 43.7 & $*$ \\
\hline $\begin{array}{l}\text { 1st L. } \\
\text { 2nd L. }\end{array}$ & $2 \times 2$ & - & 23.1 & - & 45.2 & 66.4 & - & 45.9 & $*$ \\
\hline $\begin{array}{l}\text { 1st L. } \\
\text { 2nd L. }\end{array}$ & $2 \times 2.5$ & - & 22.7 & - & 49.7 & 84.6 & - & $*$ & $*$ \\
\hline $\begin{array}{l}\text { 1st L. } \\
\text { 2nd L. }\end{array}$ & $2.5 \times 2.5$ & - & 27.4 & - & 53.6 & 98.1 & - & $*$ & * \\
\hline
\end{tabular}

Stars indicate the sets where the demand is higher than the pump capacity.

Two different scenarios of adjusting the wiper speed have been investigated. In the first scenario, the wiper activity is adjusted according to the visibility through the front screen, which is done completely manually by a driver. The manual adjustment of the wiper activity is applied by a person sitting in the driver's seat and the front visibility is adjusted by the clear view of lamps placed on the front wall, a similar condition to car's rear lights on the streets. This means, an individual person decides when to apply each single wipe, depending on the front visibility. In the second scenario, an automatic wiper speed adjustment option is used that considers different sensitivities. It's worth mentioning that the sensitivity settings can change from one car to another. This depends on the specific protocols implemented by the manufacturers. Different sensitivities are mainly defined for drivers' comfort in different precipitation conditions. In this case, the wiper system controls the adjustment of the wiper activity. The analysis concerning wiper frequency is solely carried out for one stationary car here which does not move under the rainfall simulator. In reality, the wiper speed could change for the same rain intensity depending on the car type, car speed, rain type, and windshield angle. It should be noted 

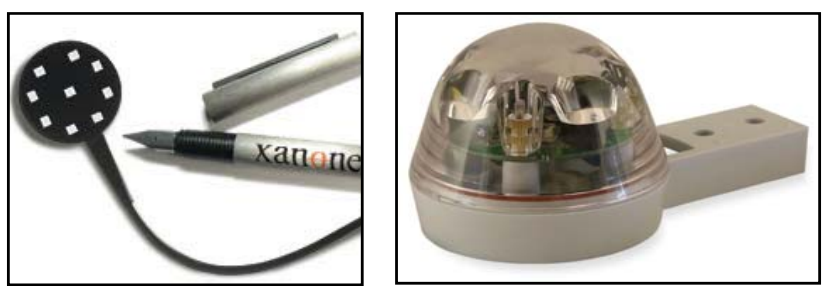

Fig. 2. Optical sensors, left: Xanonex, and right: Hydreon (Hydreon, 2012; Xanonex, 2012).

that different car types have different dimensional characteristics which may influence the aerodynamics of the raindrops and, accordingly, the sensor readings. Besides, different cars have specific wiper systems which lead to dissimilar classes of wiper frequency. According to the functionality of the optical sensors measuring the rainfall (i.e., change in beam intensity), any foreign object passing the optical sensor might have influence on the signals coming from the device. It is thus important to mention that in automatic wiper systems, each time the wiper cleans off the windshield it passes in front of the optical sensor, which may affect the signals coming from the optical sensors controlling the wiper speed. However, in practice this noise could be filtered out because of a similar effect on the signals every time the wiper blade passes the optical sensor.

\subsubsection{Optical sensors}

As alternative to the wipers, the utilization of optical sensors as measurement devices which are available on modern cars for automating the wiper activities is investigated here. Two optical sensors have been employed in this study for measuring rainfall intensity. The output of the sensors is a function of the amount of water sensed on the surface of the device. The functionality of the two devices is similar, but the output is different. The two optical sensors are presented in Fig. 2.

The Hydreon sensor (Hydreon, 2012) is fully calibrated by the manufacturer and ready to be used for different purposes, for example, measuring rainfall, wiper control on the vehicles, irrigation control, etc. This device is capable for multipurpose use and is, according to the specification, able to sense raindrops smaller than half a millimeter. The device bounces infrared beams within its lens. The effect of drops on the surface allows some of the beams to escape. This can be explained by the principles of light refraction. The change in beam intensity is considered as an indication of rain amount on the surface. Here, each sensor reading corresponds to $0.01 \mathrm{~mm}$ of rainfall. Figure 3 illustrates how the Hydreon works. As can be seen, a raindrop on the surface results in escaping some beams and, accordingly, changing in beam intensity.

The Xanonex sensor (Xanonex, 2012) is especially made to be used on cars for automating the wiper activities. For this purpose, it can be attached directly to the front windshield.

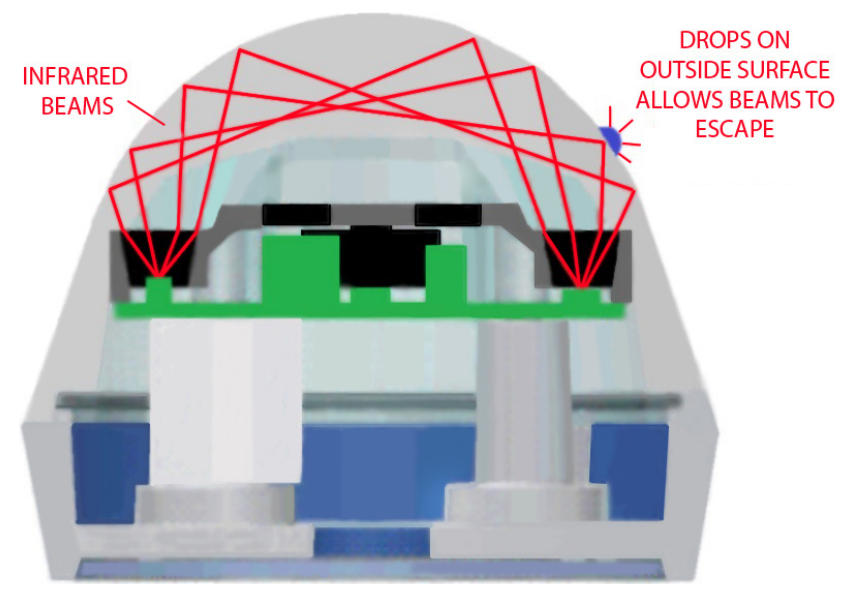

Fig. 3. The functionality of the Hydreon sensor (Hydreon, 2012).

This device works in a similar way as the Hydreon. Eight LEDs placed in a circle and the sensor in the center form the main parts of the device. The LEDs emit infrared beams out of the device. Depending on the water amount on its surface, which acts as obstacles on the windshield, part of the emitted beam is reflected back to the sensor. In principle, the sensor is implemented in an electrical circuit where a direct current flows and the flow is blocked for a certain time. This blockage appears as a signal length, which is a function of water amount. Here, the accumulation of the signals over a minute $\left[\mathrm{s} \mathrm{min}{ }^{-1}\right]$ is analyzed.

According to the sensing principles of the devices, it is postulated that the rainfall measured by the optical sensors is solely a function of water amount on the sensors' surfaces. As a result, it is assumed here that the droplet size distribution of the artificial rainfall is not relevant for measuring the correct rainfall intensity by the optical sensors.

\subsection{Car speed simulator}

One of the main influencing factors on the estimation of rainfall by a car is its speed. Analyzing an object with a certain velocity under rain has been investigated by physicists and other scientists. Bocci (2012) has proven that the amount of water hitting an object under rain depends on its shape, its orientation, wind direction and rain intensity. The main purpose of the car speed simulator is to investigate this effect in the laboratory.

Figure 4 shows the rotating machine which has been used in the laboratory. The electrical motor of the machine is able to work with different speeds and as a result simulate car speeds. Two optical sensors are placed on a rotating machine. In order to simulate an average windshield angle, the Xanonex is placed at an angle of $45^{\circ}$. By changing the rotational speeds $(\omega)$ in the car speed simulator, different tangential speeds $(u)$ are produced: 


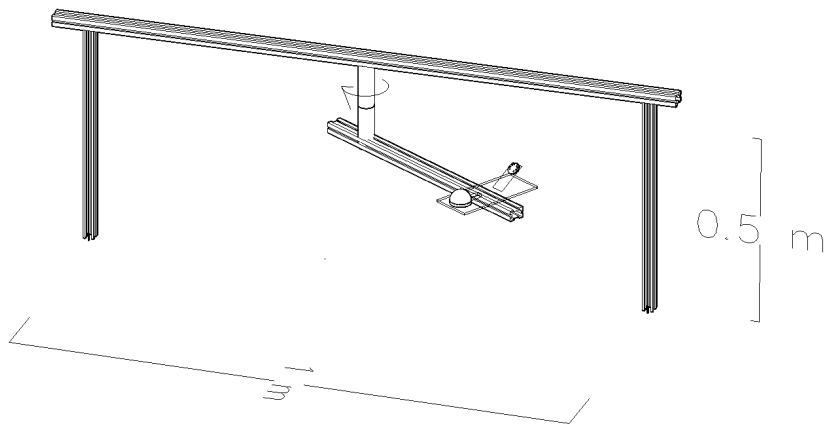

Fig. 4. Rotating machine used to simulate car speed, with two optical sensors placed on the device.

$u=r \omega$,

where " $r$ " is the radial distance, which is constant.

In order to measure the rotational speed of the device, a digital laser based tachometer with the stated accuracy of $\pm 0.05 \%+1$ digit is used.

It is necessary to mention that the devices used here are under the rain without being cleaned off by the wiper system, unlike when implemented on cars with the wiper system cleaning off the droplets on the sensors repeatedly.

The experiments with the car speed simulator are carried out separately from the experiments for deriving the $W-R$ relationships. For each individual run, the dynamic sensors are compared with the static ones of the same type. Speeds of up to $45 \mathrm{~km} \mathrm{~h}^{-1}$ are reached and tested.

The estimation of rainfall is affected by different factors including (a) the horizontal angle of the optical sensor which is representing the windshield angle, (b) the rain droplet velocity, and (c) the car speed. The rain droplet velocity can be interpreted as an indicator for the rain type. Considering the direction of the moving plane (car) as the $x$ axis and the direction of the falling rain drops as the $z$ axis, the windshield angle affects the projected area corresponding to both axes.

Bocci (2012) introduced $\boldsymbol{v}=\left(v_{x}, v_{y}, v_{z}\right)$ as the rain velocity where the vertical component, $v_{z}$, depends on the drop size. He called $\rho$ the ratio between the mass of water drops that are found within a given volume and the volume itself. Afterwards, he defined the rain density as vector:

$\boldsymbol{j}_{0}=\rho \boldsymbol{v}$.

He then introduced a vector for the moving objects, considered as a plane, representing the velocity $\boldsymbol{u}=(u, 0,0)$. Subsequently, for the moving objects, an apparent rain density $\boldsymbol{j}$, which differs from $\boldsymbol{j}_{0}$ can be defined:

$\boldsymbol{j}(u)=\rho(\boldsymbol{v}-\boldsymbol{u})=\rho\left(v_{x}-\boldsymbol{u}, v_{y}, v_{z}\right)$.

He proposed the following equation representing the rain flux as the surface integral over the rain density $j$ :

$\Phi(u)=\oint_{S} j \mathrm{~d} A$.
Restricting the integration to the "wet surface" of the object, the rain flux is defined as

$\Phi(u)=\oint_{S_{\mathrm{w}}}|j \mathrm{~d} A|$.

Assuming always vertical rainfall (no horizontal effect of the wind, $v_{x}=0$ and $v_{y}=0$ ) and $\theta$ as the windshield angle, the ratio between the rain flux observed by the dynamic device and static device becomes

$$
\begin{aligned}
\eta & =\frac{\Phi_{\text {dynamic }}(u)}{\Phi_{\text {static }}(u)}=\frac{u_{x} \cdot A \cdot \sin (\theta)+v_{z} \cdot A \cdot \cos (\theta)}{v_{z} \cdot A} \\
& =\frac{u_{x} \cdot \sin (\theta)}{v_{z}}+\cos (\theta) .
\end{aligned}
$$

This theoretically obtained ratio $\eta$ will be compared later against the empirically obtained results from the experiments with the rotating machine.

\subsection{Data processing}

The data from the dynamic optical sensors are transmitted using a wireless connection. Processing of the data by a single $\mathrm{PC}$ requires no further synchronization. In order to process the data from the tipping bucket and optical sensors, free data logger software (HTerm) has been used (Hammer, 2006).

\section{Analysis of the produced rainfall}

\subsection{Homogeneity of the produced rainfall}

The measurement devices are placed under the rain simulator at different locations. Since the rain amounts on these points are compared, the homogeneity of the produced rainfall needed to be investigated. The homogeneity of the rainfall produced in the laboratory is verified with the help of 48 beakers. They are symmetrically placed at a distance of $50 \mathrm{~cm}$ from each other. For each individual setting of the rain simulator, the amount of water kept in each of the beakers after each run is measured. Figure 5 shows an example of the water depth distribution for a pressure of 2 bars and the nozzle combination class 6 in Table 2 .

It shows that the water amount kept in the beakers at the two locations, where tipping bucket and the optical sensors are located, is not identical but very similar (48 and $50 \mathrm{~mm}$, respectively). However, the two optical sensors receive the same amount of water because of their proximity. The water depth distribution varies between the different cases of nozzle combinations and pressures. In order to assess the error resulting from non-homogeneous rainfall distribution the relative deviation in water depth between the two points at the locations of the tipping bucket and the optical sensors is calculated as follows: 


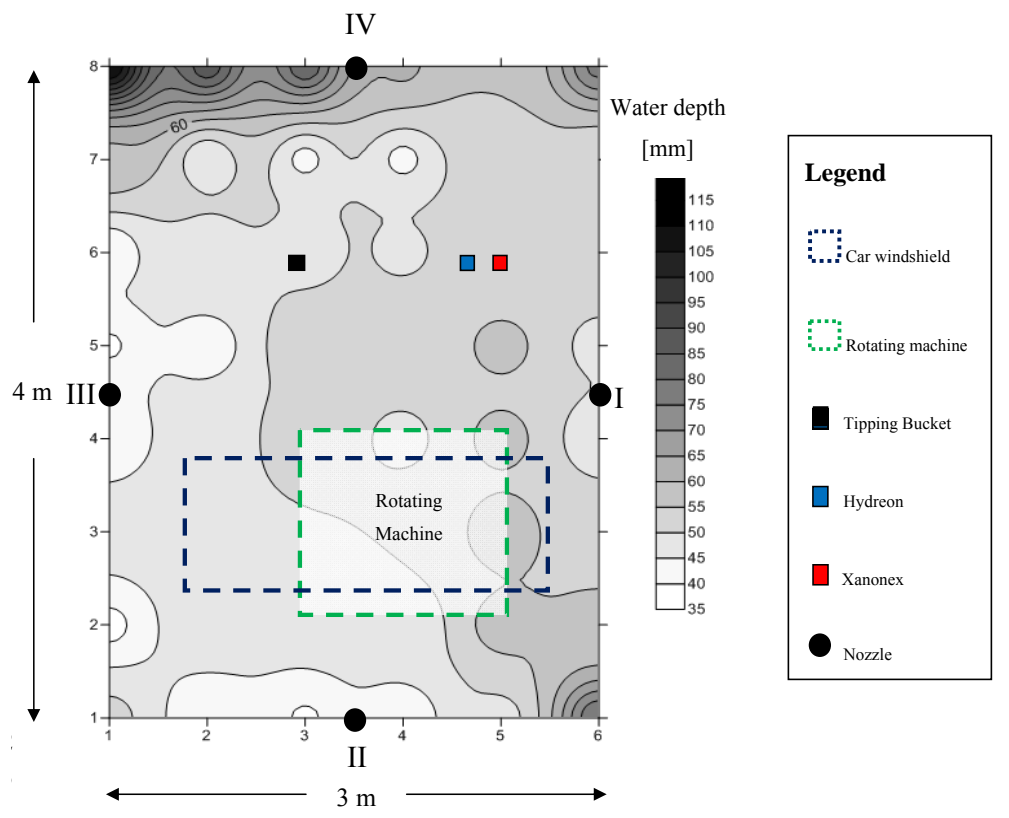

Fig. 5. Distribution of the accumulated rainfall depth over the sprinkler area for the case of nozzle combination class 6 with 2 bar pressure and the permanent location of measurement devices.

RDev $=\frac{x_{\text {tipp }}-x_{\text {opt. sensors }}}{x_{\text {tipp }}} \times 100$.

Table 4 shows statistical information of the produced rainfall for a selection of 21 cases of different pressures and nozzle combination classes. The rainfall amount at the points on the edges of the sprinkler area is much higher than at the inner points because of the proximity of these points to the nozzles and the wall. For this reason, the statistics in Table 4 are calculated without considering these outer points, including only the 24 inner measurements.

Considering the total sprinkler area covered by those 24 beakers the rainfall distribution is still quite inhomogeneous as shown especially by the coefficient of variation (CV) in Table 4. Although the design of the rain simulator is based on 2 bar pressure, a pressure of 2 bars does not always provide the most homogeneous distribution. For example, a pressure of 1 bar in class 1 provides a more even rainfall depth distribution, whereas a pressure of 2 bars in class 5 provides more homogeneous rainfall than the other two pressures.

However, in order to assess the influence of the rainfall distribution on the $W-R$ relationships the relative deviation RDev between the measurement points is relevant. Positive values of the RDev illustrate the situations in which the water depths in beakers at the tipping bucket location are larger than in beakers at the locations of the optical sensors, and vice versa. For example, the relative deviation RDev for class 1 at a pressure of 1 bar is about $0.0 \%$ meaning that the amount of water kept in the beakers at the two points is identical, while at a pressure of 2 bars RDev is $21.1 \%$ meaning more water has been kept in the beaker where the tipping bucket stands than in the beaker where the optical sensors are located. The average value of all the estimated relative deviations is $-5.8 \%$. This average error is most relevant to assess the influence of the non-homogeneous rainfall distribution on the estimation of the $W-R$ relationships. A mean relative deviation of about $-6 \%$ in rainfall depth between reference and sensor locations is assumed to be acceptable and to have only little influence on estimation of the $W-R$ relationships.

\section{2 $W-R$ relationship}

\subsubsection{Wiper frequency}

First, the initial idea of considering wiper speed as an indicator for the rain intensity is investigated. Figure 6 shows the results of a linear regression for the $W-R$ relationship of a Ford SMAX automobile with automatic wiper system where a tipping bucket gauge is taken as the reference device. Each point illustrates an individual run lasting between 10 and $15 \mathrm{~min}$. The wiper speed is adjusted either completely manually (Fig. 6, left panel) or automatically (Fig. 6, right panel). The dashed lines illustrate the $95 \%$ prediction limits for the prediction of an individual observation. Because of technical constraints and restrictions in using all the nozzles when placing the car, depending on its dimension, it was not possible to apply all the cases. As a result, the number of points in Fig. 6 differs from the number of possible runs provided in Table 3. The same is valid for the automatic wiper adjustment when the highest wiper frequency (when not moving) 

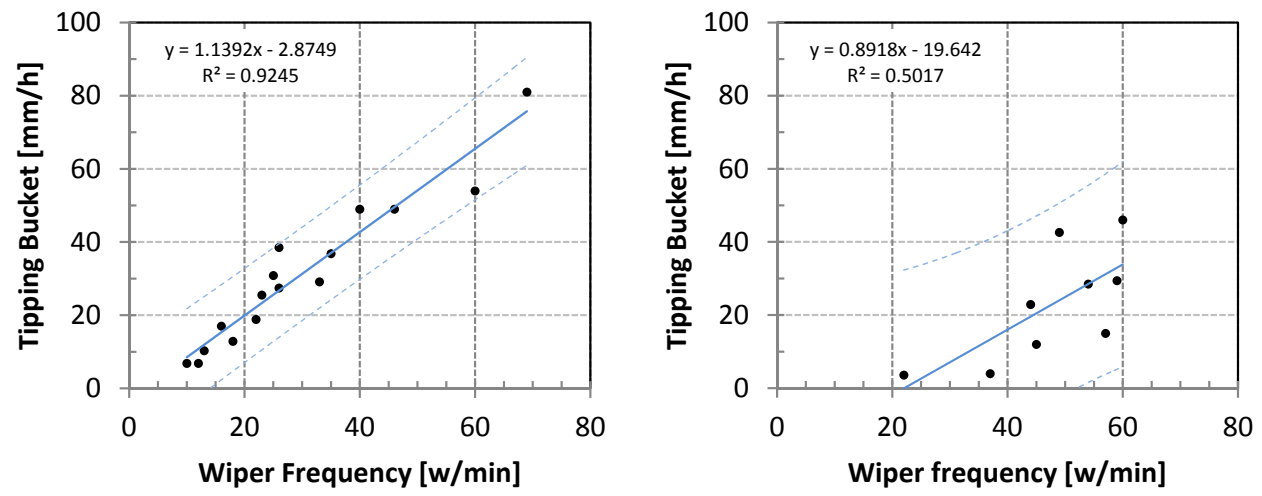

Fig. 6. Relationship between wiper frequency $(W)$ and rainfall intensity $(R)$ using manually (left panel) and automatically (right panel) adjusted wiper activities and the tipping bucket as the reference using a Ford SMAX as test car.

Table 4. Homogeneity statistics related to 21 cases of nozzle combination and pressures applied in the laboratory experiments.

\begin{tabular}{|c|c|c|c|c|c|}
\hline Class & $\begin{array}{c}\text { Pressure } \\
\text { [bar] }\end{array}$ & $\begin{array}{l}\text { Mean } \\
{[\mathrm{mm}]}\end{array}$ & $\begin{array}{r}\text { Std dev } \\
{[\mathrm{mm}]}\end{array}$ & $\begin{array}{l}\mathrm{CV} \\
{[\%]}\end{array}$ & $\begin{array}{r}\mathrm{RDev} \\
{[\%]}\end{array}$ \\
\hline \multirow{3}{*}{ Class 1} & 1 & 9.25 & 3.48 & 37.6 & 00.00 \\
\hline & 2 & 13.42 & 6.85 & 51.0 & 21.10 \\
\hline & 2.5 & 17.46 & 7.82 & 44.7 & -35.00 \\
\hline \multirow{3}{*}{ Class 2} & 1 & 16.58 & 5.87 & 35.4 & -45.00 \\
\hline & 2 & 32.04 & 8.75 & 27.3 & -14.30 \\
\hline & 2.5 & 39.54 & 6.14 & 15.5 & -25.00 \\
\hline \multirow{3}{*}{ Class 3} & 1 & 26.67 & 7.84 & 29.3 & 25.90 \\
\hline & 2 & 35.79 & 8.80 & 24.5 & -25.00 \\
\hline & 2.5 & 46.04 & 12.72 & 27.6 & -6.70 \\
\hline \multirow{3}{*}{ Class 4} & 1 & 31.04 & 5.09 & 16.3 & -11.40 \\
\hline & 2 & 57.21 & 10.31 & 18.0 & -15.70 \\
\hline & 2.5 & 61.79 & 10.28 & 16.6 & -2.60 \\
\hline \multirow{3}{*}{ Class 5} & 1 & 37.08 & 6.26 & 16.8 & -22.60 \\
\hline & 2 & 65.13 & 8.27 & 12.6 & 31.10 \\
\hline & 2.5 & 65.42 & 9.66 & 14.7 & 4.80 \\
\hline \multirow{3}{*}{ Class 6} & 1 & 31.21 & 6.93 & 22.2 & -20.00 \\
\hline & 2 & 49.63 & 5.28 & 10.6 & -4.20 \\
\hline & 2.5 & 55.29 & 6.65 & 12.0 & 8.60 \\
\hline \multirow{3}{*}{ Class 7} & 1 & 46.63 & 6.01 & 12.8 & 0.00 \\
\hline & 2 & 61.88 & 9.89 & 15.9 & 10.30 \\
\hline & 2.5 & - & - & - & - \\
\hline \multirow{3}{*}{ Class 8} & 1 & 66.25 & 8.39 & 12.6 & 2.50 \\
\hline & 2 & - & - & - & - \\
\hline & 2.5 & - & - & - & - \\
\hline
\end{tabular}

is $60 \mathrm{w} \mathrm{min} \mathrm{m}^{-1}$ ("w" represents the number of wipes). Here, the experiment is stopped after reaching this threshold.

There are different sensitivities defined for the automatic wiper system in this car, only one of the higher sensitivities is illustrated here.
Apparently, a relatively strong relationship exists between rainfall intensity and wiper speed for the manual adjustment. The result of the wiper activity adjustment, according to front visibility, supports the initial idea of considering wiper speed as an independent variable in the $W-R$ relationship. The relationship between automatic wiper frequency adjustment and rainfall intensity shows much weaker correlation and higher uncertainty. Reasons for that may be (a) too simple data processing of data readings from the optical sensor controlling the wiper activity, and (b) the point measurement of the optical sensor which may not be representative for the total water amount on the windshield.

Apart from the better suitability of the manual wiper adjustment for the establishment of a $W-R$ relationship, it may be concluded that the manual adjustment of the wiper speed is superior for drivers when compared to the automatic wiper system. So, it may be expected that advancements in the development of automatic wiper systems will provide better $W-R$ relationships in the future. With the current sensors, the driver has to adjust the sensitivity manually for different conditions to have optimal front visibility. This should not be the case if the automatic wiper control system works optimally.

Here, at first a linear $W-R$ relationship is assumed for all the analyses. However, since the lines do not pass the origin, the relationship between the two variables may be nonlinear especially for small intensities.

\subsubsection{Optical sensors}

Figure 7 shows the $W-R$ relationships between the data readings from the optical sensors and the rainfall intensity measured by the tipping bucket. Each point in this figure represents one individual run lasting between 10 and $15 \mathrm{~min}$; the dashed lines illustrate the $95 \%$ prediction intervals. Although the Hydreon sensor was considered as calibrated, Fig. $7 \mathrm{~b}$ shows an underestimation of the rainfall by this device. However, the high coefficient of determination $\left(R^{2}\right)$ shows that this underestimation could be interpreted 

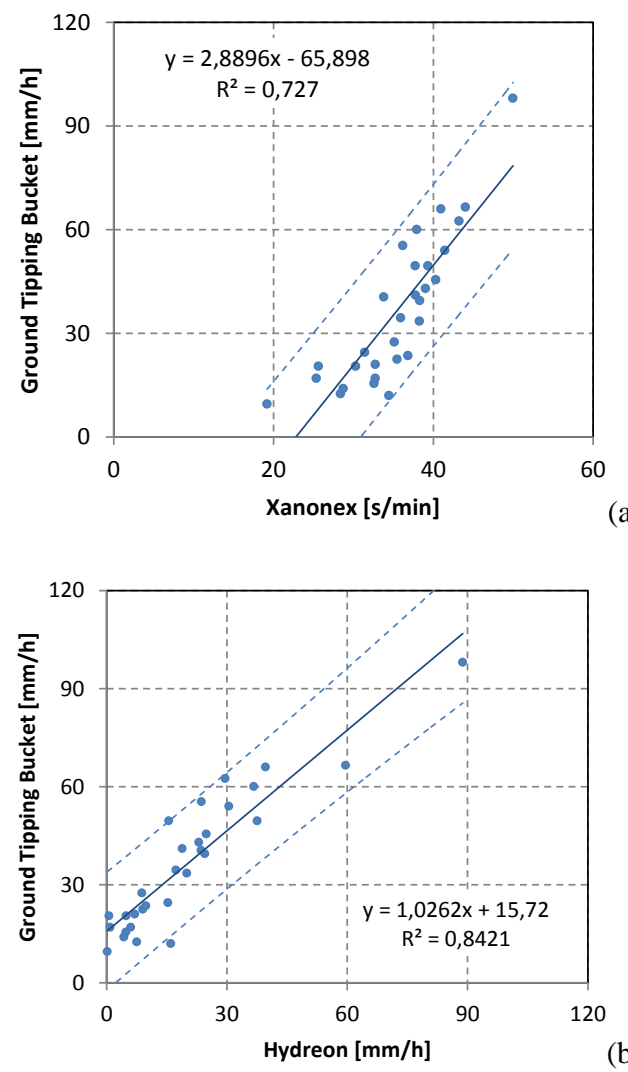

(b)

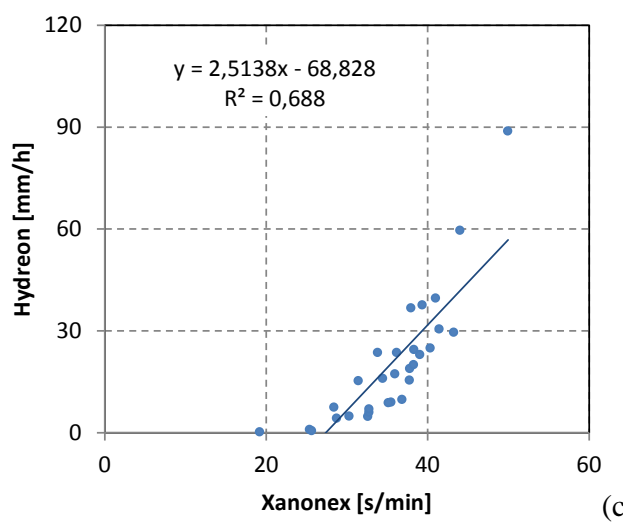

Fig. 7. Comparison of the optical sensors with the reference device, tipping bucket, with $95 \%$ prediction limits.

as a systematic error which may be corrected by recalibration. The relationship between the data readings from the Xanonex optical sensor and the rainfall intensity from tipping bucket shows lower $R^{2}$ value (Fig. 7a) compared to the Hydreon. A possible reason for the lower $R^{2}$ value and the concentration of the data readings in the range between 20 and $40\left[\mathrm{~s} \mathrm{~min}^{-1}\right]$, Fig. $7 \mathrm{c}$ ) might be the nonlinear relationship between the signal lengths and measured rainfall intensities. The higher $R^{2}$ value for the Hydreon in comparison to Xanonex may also be due to a better calibration or a better suitability of the device. The correlation between the data readings from the two optical sensors (Fig. 7c) is not as strong as the former two. This shows the difference in the calibration procedure of the devices as well as their sensitivities.

The similarity of the derived $W-R$ relationships for the automatic wiper adjustment (Fig. 6, right panel) and the Xanonex (Fig. 7a) shows the likely comparable data processing in both cases (i.e., a possible similar principle to the derived $W-R$ relationship). It can be concluded that a better calibration (e.g., considering nonlinear relationship) for the optical sensors controlling the automatic wiper systems may improve the performance of the system resulting in more convenient automatic wiper system for drivers.

\subsection{Car speed simulator}

Car speed is one of the important influential factors for the estimation of rainfall by moving cars. Theoretically, there is a positive linear relationship between the velocity of an object with a plane surface under rain and the water mass hitting the object (Bocci, 2012). This means when a car moves with higher speed the rainfall intensity measured by car sensors would be overestimated compared to a stationary ground reference value, linearly proportional to its speed.

Figure 8 illustrates the results of the car speed simulator in the laboratory. This does not involve using a car but the car speed simulator (see Fig. 3). The black line represents the mean ratio of the sensor readings from the dynamic and static device $\eta$ (Eq. 6) versus the sensor speed in different rainfall intensities. The gray area shows the range between the upper and lower quartile considering 22 runs with different rainfall intensities. Apparently, the ratios derived in the laboratory are not linear and have a tendency to become constant after a certain speed. There may be three reasons explaining this behavior: (a) the shielding effect of the remaining drops after a certain speed, which introduces a hypothetical capacity for the sensor's surface (i.e., the accumulated drops may prevent the incoming drops from affecting the sensor readings); (b) the centrifugal force on the drops, which draws the remaining drops from the center of rotation and may cause noises in the sensor readings; and (c) the special aerodynamics of the small plane carrying the optical sensor in the laboratory experiments (see below). Assuming that the first linear part of the measurements (Fig. $8 \mathrm{~b}$ up to $20 \mathrm{~km} \mathrm{~h}^{-1}$ ) is correct; a linear extrapolation would provide the complete relationship which may be applied also for higher speeds.

It has been discussed that the ratio $\eta$ (Eq. 6) between the dynamic device and the static device dependents on (a) rainfall velocity $\left(v_{z}\right)$, (b) the horizontal angle $(\theta)$, and (c) the object speed $\left(u_{x}\right)$. The rain drop velocity could be interpreted as the rain type. Lull (1959) has shown that there is a strong relationship between rain type and fall velocity; usually the higher the fall velocities, the heavier the rain. In his classification, velocities from 0.003 to $7.9 \mathrm{~m} \mathrm{~s}^{-1}$ cover the rain types from fog to extreme rain. Using Eq. (6) with $\theta=45^{\circ}$, the blue 

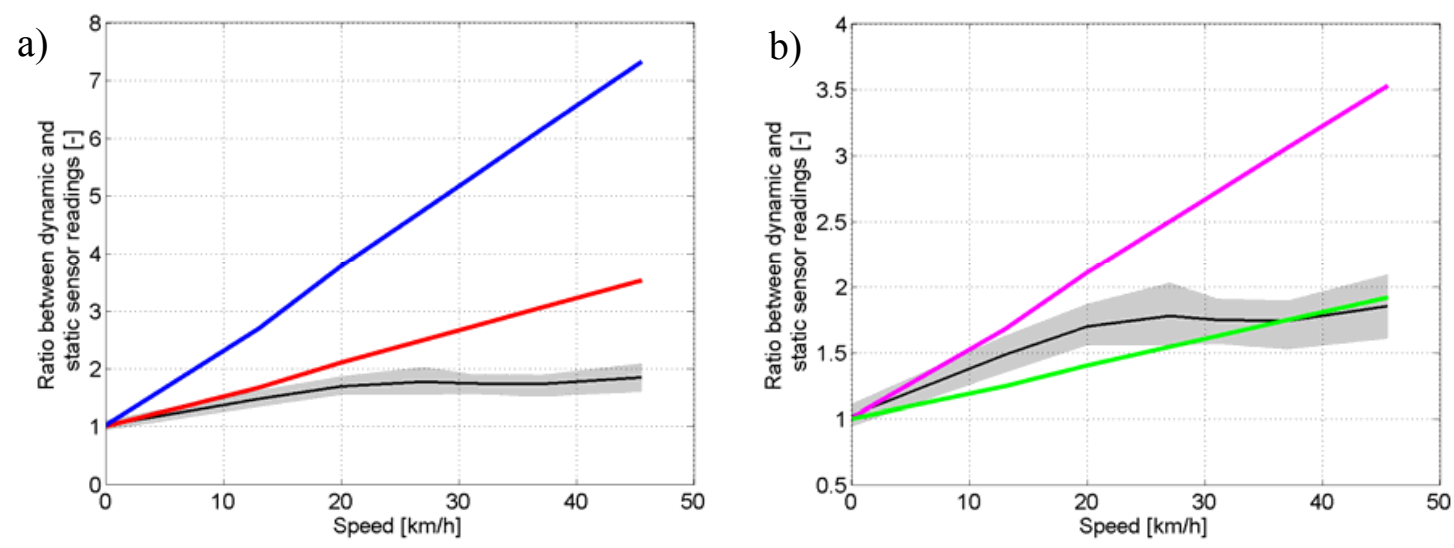

Fig. 8. Black line: experimental results of the car speed simulator with gray uncertainty boundaries. (a) theoretical ratios for assumed rainfall velocity of $2 \mathrm{~m} \mathrm{~s}^{-1}$ (blue) and $5 \mathrm{~m} \mathrm{~s}^{-1}$ (red). (b) theoretical ratios for assumed windshield angle of $70^{\circ}$ (green) and $45^{\circ}$ (purple) at an assumed raindrop velocity of $5 \mathrm{~m} \mathrm{~s}^{-1}$. The empirical relationship is derived by the car speed simulator and there is no car involved in this experiment.

line and the red line in Fig. 8a show the theoretical ratios for assumed rainfall velocity of 2 and $5 \mathrm{~m} \mathrm{~s}^{-1}$, respectively.

Due to the fact that the rainfall produced in the laboratory is from the nozzles with a height of $3 \mathrm{~m}$ only, the terminal velocity of the raindrops is lower than natural events. Knowing this, the black line in Fig. 8 should have a steeper slope compared with red and blue lines representing natural rain with higher velocities. This uncharacteristic behavior may be explained by aerodynamic effects. That is the small plane used in the laboratory may not receive all drops in the air volume in front, but several drops may be blown away.

The windshield angle is a factor which influences the rainfall estimation by the moving cars. By assuming the raindrop velocity at $5 \mathrm{~m} \mathrm{~s}^{-1}$ in Eq. (6), the green line and the purple line illustrate the effect of the angle on the ratio corresponding to an angle of $70^{\circ}$ and an angle of $45^{\circ}$ in Fig. 8b, respectively. Note that the black lines and the gray range in Fig. 8a and $b$ represent the same data; the only difference between the two figures is the scaling of the $y$ axis.

Figure 8 indicates also that the effect of rain type, in terms of rainfall velocity, on the overestimation of rainfall is likely larger than the influence of the windshield angle.

\section{Summary and conclusions}

The feasibility of considering moving cars as rain gauges to estimate areal rainfall is discussed in theory using computer experiments by Haberlandt and Sester (2010). The main objective of this study was to develop a relationship between sensor readings $(W)$ and rain rate $(R)$ based on laboratory experiments to quantify the errors. Therefore, a rainfall simulator with the ability to produce a wide range of rain intensities is designed and constructed. Analyses of the rainfall produced in the laboratory are accomplished using a tipping bucket as reference device. Two variables were considered as sensor readings in this study: wiper speed, and readings from two optical sensors which can be placed on cars to automate wiper activity. The use of wiper speed as an indicator for the rain intensity is investigated by adjusting the wiper speed either completely manually, which is executed by a person and might be subjective depending on the person in charge, or automatically. Additionally, the influence of the car speed on the estimation of the rainfall is investigated with the help of a car speed simulator.

The results of this investigation can be summarized as follows:

1. The result of the manual wiper activity adjustment, according to front visibility, shows a strong relationship between rainfall intensity and wiper speed. This supports the initial idea of considering wiper speed as the main independent variable in the $W-R$ relationship.

2. The derived $W-R$ relationship between automatic wiper frequency adjustment and rainfall intensity shows weaker correlation and higher uncertainty. Possible reasons for that include data processing of the readings from the optical sensor and the point measurement of the optical sensors controlling the wiper activity.

3 . In addition to wiper activity analyses, the $W-R$ relationship has been derived for optical sensors. The Hydreon sensor was considered as calibrated, but an underestimation of the rainfall sensed by the device has been observed. This underestimation may be interpreted as a systematic error considering a relatively strong $W-R$ relationship for the Hydreon and the low relative deviation between the sensor and the tipping bucket. The derived $W-R$ relationship for the Xanonex is weaker. Due to the narrow range of the data readings 
and also a large (non-zero) intercept in the $W-R$ relationship, better calibration of the device may lead to better $W-R$ relationships.

4. The similarity of the derived $W-R$ relationship for automatic wiper adjustment and the Xanonex optical sensor shows possible similarity in data processing for both cases. It can be concluded that a better calibration of the optical sensor controlling the wiper activities may improve the $W-R$ relationship as well as the performance of the automatic wiper system for drivers.

5. A positive relationship between the velocity of the optical sensor located on the car simulator under rain and the water mass hitting the sensor has been observed. Theoretically, a positive linear relationship exists between the two criteria, but in the laboratory the results are only approximately linear up to a speed of about $20 \mathrm{~km} \mathrm{~h}^{-1}$ and become almost constant after that. Assuming that the first part of the function is correct, a linear extrapolation would provide the complete relationship which may also be applied for higher speeds.

6. Interpreting the drop velocity as the rain type, it has been observed that the effect of rain type on the overestimation of rainfall is larger than the influence of the windshield angle. This means that by knowing the drop velocity, the rainfall overestimation could be corrected more accurately.

Altogether, the results of the laboratory experiments have shown that it is possible to derive $W-R$ relationships from the sensor readings. However, there are many influential factors which need further investigation, for example, the aerodynamics of the plane in the car speed simulator or the droplet size distribution in the laboratory.

One limitation of this study is producing rainfall intensities only in the range from 9 to $98 \mathrm{~mm} \mathrm{~h}^{-1}$. This range starts from a quite high rain intensity, compared with natural rain events, but it is quite wide for analyzing the sensor readings from optical sensors.

Equation (6) shows that by changing the windshield angle to $0^{\circ}$, the derived ratio between the dynamic optical sensor and static optical sensor becomes 1 . This means that by placing an optical sensor completely horizontal, there would be no relative influence of the car speed on the sensor readings. Future work covers investigating an optical sensor when located horizontally in the laboratory, the influence of the droplet size distributions, different car types and other factors. Currently, field experiments are carried out to obtain $W-R$ relationships in natural conditions especially for lower rain intensities. Results of the field experiments and comparisons with the laboratory derived $W-R$ relationships will be reported elsewhere. Preliminary results and possible theoretical methods of the field experiments are investigated by Fitzner et al. (2013). The rainfall data obtained by car measurements might be used separately or more likely to be combined with other sources of rainfall observations like radar data and point measurements and need to be tested for different hydrological applications.

Acknowledgements. This research was funded by the German Research Foundation (DFG, 3504/5-1). The authors wish to thank Bastian Heinrich for programming and producing the circuits of the optical sensors and the Institute for Control Engineering of Machine Tools and Manufacturing Units, $I S W$, University of Stuttgart for designing and producing the car speed simulator. Furthermore, special thanks are due to Anne Fangmann for her comments on an earlier draft of the paper and Ehsan Mohammad Pajooh for his contribution in the laboratory experiments.

The authors are very grateful to R. W. Hut and one anonymous referee for their detailed comments which helped to improve the manuscript.

Edited by: L. Pfister

\section{References}

Bocci, F.: Whether or not to run in the rain, Eur. J. Phys., 33, 1321, doi:10.1088/0143-0807/33/5/1321, 2012.

Chandrasekar, V., Chen, H., and Maki, M.: Urban flash flood applications of high-resolution rainfall estimation by X-band dualpolarization radar network, Remote Sensing of the Atmosphere, Clouds, and Precipitation IV, Proc. SPIE, Kyoto, Japan, 8523, 85230K, doi:10.1117/12.977602, 2012.

Ciach, G. J.: Local Random Errors in Tipping-Bucket Rain Gauge Measurements, J. Atmos. Ocean. Tech., 20, 752-759, doi:10.1175/1520-0426(2003)20<752:lreitb>2.0.co;2, 2003.

de Jong, S.: Low cost disdrometer, Master thesis report, TU Delft, Delft, the Netherlands, 2010.

Diop, M. and Grimes, D. I. F.: Satellite-based rainfall estimation for river flow forecasting in Africa, II: African Easterly Waves, convection and rainfall, Hydrolog. Sci. J., 48, 585-599, 2003.

Ehret, U.: Rainfall and flood nowcasting in small catchmenst using weather radar, PhD Thesis, University of Stuttgart, Stuttgart, Germany, 2002.

Erdin, R., Frei, C., and Künsch, H. R.: Data Transformation and Uncertainty in Geostatistical Combination of Radar and Rain Gauges, J. Hydrometeorol., 13, 1332-1346, doi:10.1175/jhm-d11-096.1, 2012.

Fiener, P., Seibert, S. P., and Auerswald, K.: A compilation and meta-analysis of rainfall simulation data on arable soils, J. Hydrol., 409, 395-406, doi:10.1016/j.jhydrol.2011.08.034, 2011.

Fitzner, D., Sester, M., Haberlandt, U., and Rabiei, E.: Rainfall Estimation with a Geosensor Network of Cars Theoretical Considerations and First Results, Photogramm. Fernerkund. Geoinf., 2013, 93-103, 2013.

Haberlandt, U.: Geostatistical interpolation of hourly precipitation from rain gauges and radar for a largescale extreme rainfall event, J. Hydrol., 332, 144-157, doi:10.1016/j.jhydrol.2006.06.028, 2007.

Haberlandt, U. and Sester, M.: Areal rainfall estimation using moving cars as rain gauges - a modelling study, Hydrol. Earth Syst Sci., 14, 1139-1151, doi:10.5194/hess-14-1139-2010, 2010. 
Hammer, T.: http://www.der-hammer.info/ (last access: 25 November 2013), 2006.

Hydreon: Rain Gauge Model RG-11 Instructions, http://www. rainsensors.com/ (last access: 25 November 2013), 2012.

Javier, J. R. N., Smith, J. A., Meierdiercks, K. L., Baeck, M. L., and Miller, A. J.: Flash Flood Forecasting for Small Urban Watersheds in the Baltimore Metropolitan Region, Weather Forecast., 22, 1331-1344, doi:10.1175/2007waf2006036.1, 2007.

Lanza, L., Leroy, M., Alexandropoulos, C., Stagi, L., and Wauben, W.: WMO Laboratory Intercomparison of Rainfall Intensity (RI) Gauges - final report, IOM Report No. 84, WMO/TD No. 1304, WMO, Geneva, Switzerland, 2005.

Leijnse, H., Uijlenhoet, R., and Stricker, J. N. M.: Rainfall measurement using radio links from cellular communication networks, Water Resour. Res., 43, W03201, doi:10.1029/2006wr005631, 2007.

Lull, H. W.: Soil compaction on forest and range lands, 768, Forest Service, US Dept. of Agriculture, Washington, D.C., 1959.

Messer, H., Zinevich, A., and Alpert, P.: Environmental Monitoring by Wireless Communication Networks, Science, 312, p. 713, doi:10.1126/science.1120034, 2006.

Overeem, A., Leijnse, H., and Uijlenhoet, R.: Country-wide rainfall maps from cellular communication networks, P. Natl. Acad. Sci., doi:10.1073/pnas.1217961110, in press, 2013.

Phocaides, A.: Technical Handbook on Pressurized Irrigation Techniques, Food and Agriculture Organization of The United Nations, Rome, 2000.
Salles, C. and Poesen, J.: Performance of an optical spectro pluviometer in measuring basic rain erosivity characteristics, J. Hydrol., 218, 142-156, doi:10.1016/s0022-1694(99)00031-1, 1999.

Schilling, W.: Rainfall data for urban hydrology: what do we need?, Atmos. Res., 27, 5-21, doi:10.1016/0169-8095(91)90003-F, 1991.

Sharpley, A. and Kleinman, P.: Effect of rainfall simulator and plot scale on overland flow and phosphorus transport, J. Environ. Qual., 32, 2172-2179, 2003.

Upton, G. J. G., Holt, A. R., Cummings, R. J., Rahimi, A. R., and Goddard, J. W. F.: Microwave links: The future for urban rainfall measurement?, Atmos. Res., 77, 300-312, doi:10.1016/j.atmosres.2004.10.009, 2005.

Verworn, A. and Haberlandt, U.: Spatial interpolation of hourly rainfall - effect of additional information, variogram inference and storm properties, Hydrol. Earth Syst. Sci., 15, 569-584, doi:10.5194/hess-15-569-2011, 2011.

Vogl, S., Laux, P., Qiu, W., Mao, G., and Kunstmann, H.: Copulabased assimilation of radar and gauge information to derive biascorrected precipitation fields, Hydrol. Earth Syst. Sci., 16, 23112328, doi:10.5194/hess-16-2311-2012, 2012.

Xanonex: Xanonex Funktionsweise, http://www.xanonex.de/ (last access: 25 November 2013), 2012.

Zinevich, A., Messer, H., and Alpert, P.: Frontal Rainfall Observation by a Commercial Microwave Communication Network, J. Appl. Meteorol. Clim., 48, 1317-1334, doi:10.1175/2008jamc2014.1, 2009. 